\title{
Justified deception? The single blind placebo in drug research
}

Martyn Evans University of Wales Swansea

\begin{abstract}
"Run-in" and "washout" periods involving the withholding of medication are widely used in drug research trials in pursuit of both patient safety and scientific reliability. Such no-medication periods can be justified ethically provided that they are apparent to patients, who can thereby properly consent to undergoing them. Less widespread, but still common, is the practice of "single blinding" no-medication periods, concealing them from patients by means of placebo. Whilst all placebos involve a measure of concealment, their use is typically justified in drug research trials (i) by their preserving the uncertainty generated by the random allocation of different treatments within a drug trial; and (ii) by the researchers openly declaring both the randomisation process and the chances of receiving placebo. In the single blind placebo "run-in" or "washout", neither of these conditions is met.

This paper considers three possible defences of the practice of using single blind placebo "run-ins" or "washouts" and finds them all to fail; the practice appears ethically unjustified.

(Fournal of Medical Ethics 2000;26:188-193)
\end{abstract}

Keywords: Drug trials; single blind placebos; consent; deception; "run-ins"; "washouts"

\section{Justified deception? The single blind placebo in drug research}

It is commonplace in clinical pharmaceutical research, particularly research involving long term prophylactic medication, for the period of study of active treatment to be both preceded and followed by a "washout" period, ie a period in which no active treatment is received by the patients entered into a particular trial as subjects. ${ }^{12}$

The scientific justifications for including such periods vary somewhat according to the treatment being studied and the disease condition involved, but taken together they can be briefly summarised as follows.

The initial washout period means, first, that upon entry to the active treatment phase of the trial, a baseline assessment can be made of all the trial subjects so as to establish the extent of any disease. This extent will no longer be masked by the patient's existing prophylactic medication, all traces of which, it could be assumed, will have disappeared from the patient's body. Second, this baseline assessment can be used to establish whether the randomisation of trial subjects into different groups has yielded groups which are similar; any dissimilarities can be identified and the corresponding baseline values can be taken account of statistically when the trial results are analysed. Third, any effects of the trial medication can prima facie be attributed specifically to that medication, rather than to some unknown proportions of action distributed between the trial medicine and its predecessor. Fourth, any such effects of the trial medication can be identified by means of contrasting later clinical assessments with the baseline assessment. Fifth, the risk of unwanted, possibly harmful, interaction between the trial medication and the previous medication can be virtually eliminated.

The end-of-study, follow-up washout period can secure an end-of-trial clinical assessment that is not masked by the effects of the trial medication. Differences between this assessment and the most recent of the assessments made during the active phase will in principle assist in confirming any attribution of clinical effects to the trial medication. Moreover, the possibility is reduced or eliminated of adverse interactions when the patient's previous medication is subsequently resumed.

For the purposes of this discussion I propose to accept that these no-medication periods would indeed produce the scientific benefits claimed for them, and I propose further to accept that this constitutes a prima facie justification for instituting them.

However, a feature of a number of clinical trials is that these no-medication periods, be they run-ins prior to the active study or end-of-trial washout following it, involve the use of placebo; they are typically referred to in the reports of such studies as "single blind placebo run-in and washout periods". " This is moreover no isolated phenomenon; the inclusion of such periods has been described as routine or even ritual. ${ }^{78}$ 
To understand what is ethically troubling about this practice, we need to examine the essential characteristics of both placebos in general, and the notion of "single blinding" in particular.

Let us briefly remind ourselves of the "facts of life" concerning placebos. It would indeed be odd to tell a patient that he or she was receiving a placebo, since it is usually taken as read that the effect of a placebo depends upon certain expectations that the patient holds regarding its powers. ${ }^{9}$ These expectations in their turn depend on the patient's not knowing that he is taking what others regard as essentially inactive; in short, the expectations depend on a certain amount of concealment or deception. This concealment in itself is enough to arouse a certain amount of ethical excitement, but this is not the point I wish to pursue here. For the purposes of this discussion I shall agree that placebos may justifiably be administered in certain circumstances, including in particular those in which research subjects have agreed to be randomly allocated to different arms of a drug trial, so that they know they have a calculable chance of receiving active treatment.

\section{Coded identification}

The next point concerns the "blinding" of the placebo periods, in the present case single blinding. This means that, whilst the administration of the placebos in these washout periods was concealed (as it had to be) from the patients, it was not concealed from the investigators. Single blinding can, in practical terms, be employed at any stage of a study-provided that the active treatment is capable of being duplicated by a dummy equivalent (which is usually a simple enough matter in pharmaceutical research). Even where the allocation of patients to different treatment regimes is determined by a process of prior randomisation, all that is required is that the coded identification of each individual's treatment be available, upon inspection, to the investigators. They can thus know at any stage who has been prescribed active medication and who placebo. However, it is usual and certainly sensible for any trial protocol, in which trouble has been taken to institute randomisation, dummy medication and "blinding" of patients or subjects, to "blind" the investigators as well. For relatively little additional effort, the control of unwanted variables and the associated elimination of bias is thereby made that much more secure. ${ }^{10}$ Thus we should expect that there is a reason why parts of a trial are deliberately made single blind and not double blind.

Now in the present case there is a clear practical, indeed logical, reason. The single blind run-ins or washouts are periods in which no medication is administered; they are designed as such, and-for the scientific reasons I have reviewed above - they intentionally apply to all patients in the trial. Thus there is no distribution of patients, random or otherwise, to different regimes at these run-in or washout stages: all are treated (or rather not-treated) alike. Hence there is nothing which the investigators ought not to know (or believe) about what is administered to their individual trial subjects during these stages, making the "blinding" of the investigators both unnecessary and indeed incoherent: blinding the investigators is in these periods a meaningless exercise. But what of the patients?

The patients are receiving a placebo, and it is in the nature of things that they do not know they are receiving it: if they knew this, then it couldn't function as a placebo. ${ }^{11}$ Accordingly then they must believe either that they are certainly receiving an active drug, or-commonly enough in randomised controlled trials - that there is a definite chance that the drug they are taking is active. Unfortunately, in the case of the nomedication periods we are considering, neither of these beliefs can be true. None of them in fact has the slightest chance of receiving active treatment during the run-in or washout phases. Yet none of them realises this: the administration of placebo during the two washout phases is expressly designed to make sure that they do not realise it. They do not, in short, know that they are undergoing run-in or washout phases at all.

The problem can now be summarised thus. In clinical research concerning long term prophylactic medication, those protocols which employ single blind placebo run-ins or washouts necessarily, and intentionally, involve periods when patients on long term prophylactic medication have that medication entirely withdrawn from them, without their knowledge - and hence, it seems fair to add, without their consent. It is difficult to conceive of this other than in terms of a deliberate deception. ${ }^{12}$

\section{Moral objections}

Our moral objections can now, it seems, be summarised. One can hardly consent to be deceived, at least prospectively, without falling into paradox: if one knowingly consents, the deception is to that extent dissolved, whereas if the deception evades the consent then the consent was not truly given. And if these single blind placebo periods are indeed instituted without the trial subjects' consent, then their consent for the trial as a whole is to that extent undermined if not invalidated. 
What justification could there possibly be for such a deception?

So far as I can see, three candidates present themselves; to sustain our overall objection, we must deal with all three. We can summarise the attempted justifications simply enough. First, perhaps it is justifiable to engage in a temporary deception, which is later retrieved through the provision of a full explanation that is accepted retrospectively by the research subject. All's well that ends well, as it were, provided that on "debriefing"after the research, the research subject agrees that the deception was justified (for instance, by some greater good) and does not at that stage object to having been deceived. Second, and somewhat full-bloodedly, perhaps the deception is justified anyway by being brought under a larger calculation: the deception is scientifically necessary to the research, and as such is simply the moral price of the progress we all want. Third, perhaps the deception can be minimised and brought within acceptable limits by acknowledging the no-medication periods, but in sufficiently vague terms as to preserve the aim of protecting the research subjects from jumping to conclusions. ${ }^{13}$ Having summarised the exits, let us now try to bar them.

\section{Moral promissory note}

First, if it is true that one cannot meaningfully consent to the prospect of deception, might one be able to consent retrospectively, after the fact - and might such a consent be thought sufficiently robust as to justify the initial deception after all? That some think such a justification possible seems clear from the advice given to psychologists regarding the ethical probity of behavioural research involving deception or manipulation. ${ }^{14}$ The criterion that psychological researchers are required to employ is this (put as simply as is practicable): the investigator must in good faith have no reason to suppose that, upon subsequent disclosure of the facts of the deception, a given research subject would be so distressed or discomfited by learning what had happened to her that she would on that account wish she had not been involved in the research. If the criterion is met, if there is no other ethical impediment to the research and if, in other respects than those of the central deception, the subject can and does give a meaningful prospective consent to her association with the investigator, then the deception can proceed. The justification therefore involves a kind of moral promissory note, to be redeemed-so one hopes-when the subject is finally "de-briefed" about the deception and agrees that, retrospectively, she didn't mind. Similarly, it might well be possible to "de-brief" the subjects of studies involving single blind placebo periods after their participation is complete, having recruited them in the first place on the basis of concluding in good faith that they would not subsequently object to the deception. The immediate question facing us is therefore whether this handling of the ethics of deception offers a decent exit from the moral net we thought we had cast.

\section{Stronger complaint}

There are, I think, three ways in which we might attempt to bar this first exit. First we might complain that what I have called "retrospective consent" is inherently not robust, and should simply not be appealed to under any circumstances. This is an excessively libertarian complaint that if taken at all seriously would stand in the way of emergency-room procedures of all kinds up to and including life-sustaining surgery. Most of us would think that in these kinds of cases retrospective consent is jolly well robust enough, and on those grounds I propose to discard the complaint in its general form.

A stronger complaint is that retrospective consent is appropriate in only a limited range of circumstances, of which emergency procedures are one general kind and, perhaps, some kinds of psychological and behavioural research are another. This complaint would then be amplified by the further claim that the drug trial we have been considering does not fall within the acceptable scope of retrospective consent. The trouble here is of course that we are now obliged to say exactly why it doesn't fall within that scope-why, for instance, psychological but not physiological research can make use of it. This is not entirely easy to show. It is not shown, for instance, by appealing to the role of the manipulation of beliefs in behavioural research, because something very like the manipulation of beliefs is inherent in physiological research whenever it makes use of placebos at the active treatment stage. Perhaps it might be thought that the psychological researcher is a shrewder judge of personality and can be better relied upon than can her pharmaceutical colleagues to anticipate the reactions of her research subjects. But this seems unacceptably patronising, difficult to establish at either the general or the individual level, and in any case open to a simple solution even if it were indeed true: if you want to take advantage of a retrospective form of consent in a drug trial then simply employ a suitably skilled psychologist in the recruitment of subjects. Nor could we rule out the use of retrospective consent in physiological research on the grounds of the supposedly greater seriousness of risks 
involving physical harms. The risks of psychological harm are serious enough in much behavioural research. ${ }^{15}$ Moreover if the use of retrospective consent really is objectionable, then it seems to be because of essentially psychological rather than physical phenomena: it is not my bodily wellbeing which is harmed if you deceive me.

We have still not closed this first exit. But we can divert our quarry towards a second and quite different exit in this way: we can complain that retrospective consent is necessary only if a temporary deception is necessary in the first place, and that whilst it no doubt is necessary in some psychological research it is not necessary in the investigation of prophylactic medication. The question is not, by the way, whether the no-medication periods themselves are necessary-I conceded right at the outset the justification for including them in the trial. The question now is rather whether they really have to be secret. For if they don't, then the deception is not necessary, and if the deception is not necessary then it seems categorically unjustified: retrospective consent cannot rescue it, and the first exit will after all be closed.

\section{Moral discomfort}

The second exit relies on showing that the deception is morally necessary. This in turn seems to presuppose both that the deception is necessary to the research and that the importance of the research is sufficient to overcome the moral discomfort which the deception causes to us; in effect, the price represented in the deception is high, but not too high. This exit seems rather easier to close than the first appeared: the deception is at any rate not scientifically or clinically necessary to the research. The research can proceed perfectly well if the no-medication periods are openly declared to the patients, because none of the objectives (rehearsed above) which are secured by the no-medication periods relies upon the patients' beliefs about what medicines they are taking. Indeed "open", that is, unblinded, run-in and washout periods are commonly used in clinical pharmaceutical trials, and are naturally self-evident to the patients concerned. There is no reason to think that the importance or urgency of the condition being studied could in and of itself generate anew both the need and the justification for secrecy regarding the no-medication periods. Indeed, the reverse is more likely: the greater the seriousness of the condition the more important it becomes that patients properly consent to what happens to them in research, given that the principal aim of clinical research is to benefit not the subjects, but hypothetical future patients. ${ }^{16}$ (An alternative possibility-which I think we need raise only to dismiss - is that in the case of some psychiatric illnesses, clinical depression being prominent among them, the fact of a nomedication period would be misunderstood by and hence alarming or even dangerous to the research subjects. But such a view would confuse the aims of clinical research and clinical management; moreover the very seriousness of pharmaceutical management of psychiatric illness counts heavily against the use of no-medication periods in relevant clinical research.) In sum, therefore, since taking this second exit relies on showing that the deception is morally justified because it is morally urgent, then the exit remains closed: as we have shown, the first exit now closes with it.

There remains a third possible exit, that of attempting simply to dissolve the problem. After all, since a certain amount of concealment is tolerated in the ordinary use of placebos as controls (or, for that matter, in the use of blinding as such) then the deception which so offends us in this trial can be replaced instead by vagueness. That is, the investigators could indeed secure consent for an openly declared washout phase, but simply without specifying when it would occur. A stratagem of this sort is proposed by Ramsay, defending the exceptional use of placebo run-ins:

"[T]he informed consent must be appropriate. One form of words might be: 'During this study there will be one or more periods during which you will have inactive (placebo) treatment. It is important for the success of the study that you are unaware which study periods these are'."

Ramsay does not spell out how the stratagem would be justified, and the stipulation that any associated consent process must be "appropriate" is a qualification rather in need of being spelled out. We may imagine, however, that the justification might run as follows. In consenting to unspecified no-medication periods, patients would not have been deceived because they would not have been induced to believe anything that was both specific and false: they could be told, truthfully, that one or more no-medication phases would occur for all patients "at some stage" in the trial. They could also be told - though this clearly requires more than Ramsay's suggested form of words - that it would occur at the same time for all patients, and that no randomisation was operative in that phase. And they could even be told, again truthfully, that the purpose of placebo medication at that point was simply to make sure that they did not know when they were in the no-medication phase. This is, surely, as legitimate a use of placebo as any other? The placebo run-in thus 
may hope to brandish a sufficient display of virtue to force a breakout through this third exit.

But I think even this attempt will fail, and our net will close. For the new admissions resemble the old silences in being designed to avoid the reasons for concealing the washout phase. To begin with, it is not at all the case that this use of placebo is as legitimate as any other. The standard uses of placebo rest on scientific justifications, internal to the conduct and design of controlled comparative research, and based on the epistemic necessity of eliminating confounding variables. Their legitimacy is grounded in scientific necessity. But the present use of placebo has no such justification. Ramsay admits as much:

"Any blinding in the run in phase is so fragile as not to be blind at all, and I would not argue strongly between no treatment, no-matching treatment, or matching placebo. The main value of placebo treatment is perhaps to familiarise all concerned (patients, investigators, pharmacists, etc) with the procedures that are to be used throughout the study."

This use of placebo is effectively an instrument of clinical management and as such, without being the object of a specific consent, it is hard to see how it can be justified in the research context. Furthermore, the vagueness of the new admissions obscures the duration of the no-medication periods. The importance of this is related to the treatments amongst which patients will be randomised in the active treatment period of the study. If all the comparative arms of the study involve receiving some active treatment-as is nowadays usual - then Ramsay's suggested form of words is more or less self-defeating. Proper consent procedures for the study as a whole will make it plain that the active treatment phase involves treatment for all, and hence cannot be one of those periods in which, as Ramsay puts it, "you will have inactive (placebo) treatment". Placebo will evidently be confined to the run-in and/or washout phases, and therefore will deceive nobody; consequently Ramsay's supposition that "It is important for the success of the study that you are unaware which study periods these are" is in these circumstances a vain hope. If on the other hand the active treatment period of a study includes a placebo-only arm, as it occasionally does, ${ }^{17}$ then a separate problem arises. For now some patients will be randomised to receiving nothing but placebo for the entire duration of the study: single blind run-in, double blind treatment phase, and single blind end-of-study washout. In this instance a blanket statement to the effect that patients will have only placebo for "one or more periods" during the study is technically true, but highly misleading. Candour requires a further statement to the effect that some patients will receive no active treatment whatever during the entire study. It might be difficult to recruit patients to such a study; connectedly it might be difficult to explain to patients why their long term medication is both important to them, and yet amenable to a substantial interruption, typically of several months.

On this precise point we must remind ourselves that, whatever the purpose of single blinding the run-in and washout periods of the study, the only purpose of administering placebo in those periods is to achieve this "blinding" of the patients involved. With this in mind we ought to notice that one potential purpose for single blinding which suggests itself here would be wholly unjustified-namely that the placebo run-in be instituted in order to conceal from a patient the fact of the prolonged interruption to his or her treatment. I do not suggest this purpose ever actually obtains: I simply emphasise that it could not be justified.

We can now conclude that the third putative exit, from the moral net we have cast around the single blind placebo, is also closed. Our fish, then, is caught, albeit with some effort. The problem I have been discussing here is an unusual one, but none the less important given the large and increasing proportion of pharmaceutical clinical research devoted to investigating (particularly in the areas of asthma and hypertension) long term prophylactic agents. The use of placebo-masked washouts bespeaks at best a reticence towards informing patients or at worst a lack of respect for them that is at odds with taking seriously the dignity which clinical research subjects have a right to expect.

Martyn Evans, BA, PhD, is Senior Lecturer in Medical Humanities, the Centre for Philosophy and Health Care, School of Health Science, University of Wales Swansea.

\section{References and notes}

1 Pocock SJ. Clinical trials. Chichester: John Wiley \& Sons, 1983. 2 Spilker B. Guide to clinical trials. New York: Raven, 1991.

3 Bursztyn M, Ghanem J, Kobrin I, Fidel J, Ben-Ishay D. Comparison of verapamil and captopril in elderly hypertensive subjects: results of a randomized, single blind, crossover study. fournal of Cardiovascular Pharmacology 1993;21:84-8.

4 Levine JH, Ferdinand KC, Cargo P, Laine H, Lefkowitz M. Additive effects of verapamil and enalapril in the treatment of mild to moderate hypertension. American fournal of Hypermild to moderate hyp

5 Ruddy TD, Wright JM, Savard D, Handa SP, Chockalingam A, Boulet AP. Comparison of the efficacy and safety of once-daily versus twice-daily formulations of diltiazem in the treatment of systemic hypertension. Cardiovascular Drugs and Therapy 1995; 9:413-20. 
6 Lacourciere Y, Poirier L, Lefebre J, Archambault F, Cleroux J Comparative assessment of antihypertensive efficacy of DL-nebivolol and D-nebivolol in patients with confirmed mild to moderate hypertension. Fournal of Cardiovascular Pharmacology 1995;25:619-24.

7 Senn S. Are placebo run-ins justified? British Medical fournal 1997;314:1191-3.

8 Ramsay LE. Commentary: placebo run ins have some value. British Medical fournal. 1997;314:1193.

9 Park LC, Covi L. Non-blind placebo trial: an exploration of neurotic patients' responses to placebo when its inert content is exposed. Archives of General Psychiatry 1965;12: 331-45

10 See for instance reference 1 .
11 I am here discounting the "placebo effect" to which a proportion of even an active drug's effects are attributed.

12 This point has been made by Senn: see reference 7 .

13 Such a defence has been attempted by at least one commentator: see reference 8 .

14 The British Psychological Association's ethical guidelines for psychological research involving temporary deception require that at the time of recruiting a given subject, there be grounds for supposing that that subject would retrospectively accept the deception.

15 Wadeley A Ethics in psychological research and practice. Leicester: British Psychological Association, 1991.

16 Evans D, Evans M. A decent proposal: ethical review of clinical research Chichester: John Wiley \& Sons, 1996.

17 See, for instance, the trial reported in reference 4.

\section{News and notes}

\section{Summer Seminar in Health Care Ethics}

The Summer Seminar in Health Care Ethics, an annual one-week introduction to health care ethics will be held at the University of Seattle, Washington, USA from July 31 - August 42000.

Registration is limited to physicians, nurses, social workers, chaplains, teachers and others involved in the care of patients or the education of providers.

Albert Jonsen, Professor Emeritus in the Department of Medical History and Ethics, will lead the seminar. The cost of the seminar is US $\$ 795$ for health care professionals with degrees in law or medicine and US $\$ 770$ for other health care professionals, if payment is received by June 302000 . After that date fees increase to US $\$ 845$ and US $\$ 820$ respectively.

For further information please contact: Marilyn J Barnard, Manager, Continuing Education program, University of Washington, Medical History and Ethics, Box 357120, Seattle, WA 98195-7120. Telephone: (206) 616 1864; fax: (206) 6857515 ; email: mbarnard@u.washington.edu

\section{News and notes \\ Eighth International Congress on Ethics in Medicine}

The Eighth International Congress on Ethics in Medicine will be held on November 5 - 9, 2000 in Beer Sheva, Israel. The congress is being co-sponsored by Ben Gurion University of the Negev (Beer Sheva, Israel); Beth Israe Medical Center (New York); the Center for Jewish Medical Heritage (Tel Aviv); the British Institute of Medical Ethics (London), and the Karolinska
Institute (Stockholm). The central theme, Ethics Across Cultures, Eras and Borders, will be discussed by medical personnel, ethicists and scholars from around the world.

For further information please contact: Ethics Congress, Peltours-Te'um Congress Organisers, POB 52407, Jerusalem 91520, Israel; tel: 9722648 1245; fax: 9722648 1305; email: teumcong@netmedia.net.il 